\title{
HIGH PERFORMANCE OF LOCAL MESHFREE METHOD WITH REDUCED INTEGRATION
}

\author{
WILBER VELEZ, THIAGO ARAUJO \& ARTUR PORTELA \\ Departamento de Engenharia Civil, Universidade de Brasília, Brazil
}

\begin{abstract}
The Meshfree Method with reduced integration (ILMF) is derived through the work theorem of structures theory. In the formulation of the ILMF, the kinematically-admissible strain field is an arbitrary rigid-body displacement; as a consequence, the domain term is canceled out and the work theorem is reduced to regular local boundary terms only. The moving least squares (MLS) approximation of the elastic field is used to construct the trial function in this local meshfree formulation. ILMF has a high performance in problems with irregular nodal arrangement leading to accurate numerical results. This paper presents the size effect of the irregularity nodal arrangement parameter $\left(c_{n}\right)$ on three different nodal discretization to solve the Timoshenko cantilever beam using values fixed for the local support domain $\left(\alpha_{s}\right)$ and the local quadrature domain $\left(\alpha_{q}\right)$. Results obtained are optimal for 2D plane stress problems when compared with the exact solution.

Keywords: work theorem, reduced integration, local meshless method.
\end{abstract}

\section{INTRODUCTION}

Numerical methods based in grid, like Finite Element Method (FEM), are widely used for scientific research. Grid-based methods required high quality meshes to solve fracture mechanics problems with material discontinuity, large deformation where excessive mesh distortion takes place and other situations. The meshless methods were generated with the expectation of providing more adaptive, accurate and stable numerical solutions that can deal with problems where conventional methods are not suitable [1]. Generally, their formulation is based in the weighted-residual method [2].

Different meshless methods have been developed during the last 20 years [3]. Some methods based on a weighted-residual weak-form formulation were applied in solid mechanics such as the Diffuse Element Method (DEM) [4], the Reproducing Kernel Particle Method (RKPM) [5], and the Element-free Galerkin (EFG) [6]. Other methods emerged based on local weighted-residual weak forms, such as the Meshless Local Petrov-Galerkin Method (MLPG) [7], [8], the Meshless Local Boundary Integral Equation (MLBIE) [9], the Local Point Interpolation Method (LPIM) [10], Local Radial Point Interpolation Method (LRPIM) [11], the Meshless Finite Volume Method (FVM) [12], the Rigid-Body Displacement Meshfree (RBDMF) and the Generalized-Strain Meshfree (GSMF) [13].

The Meshfree Method with reduce integration (ILMF) formulation presented by [14], the kinematically-admissible strain field is chosen as the one corresponding to an arbitrary rigidbody displacement; as a consequence, the domain term is canceled out and the work theorem is reduced to regular local boundary terms only. The Moving Least Squares (MLS) approximation of the elastic field is used to construct the trial function in this local meshfree formulation. ILMF and the popular MLPG using the MLS approximation, this fact allowing having more precise conclusions when comparing the two methods.

The Meshfree Methods were developed to solve large displacements issues that the Finite Element Methods could not handle [15]. This Meshfree approach can be implemented to solve any real engineering problems. The ILMF and MLPG presented are suitable to work with inhomogeneous materials, since it has no elements with continuous physical properties, 
and it has intrinsic ability to adapt to irregular geometries, once the nodes can be automatically generated anywhere inside and in the edges of the problem domain.

This paper is focused on the size effect of the irregularity nodal arrangement parameter $\left(c_{n}\right)$ when is used a fixed value for the local support domain $\left(\alpha_{s}\right)$ and the local quadrature domain $\left(\alpha_{q}\right)$. It presents a comparison of the energy and displacement relative error for three different irregular nodal distribution to solve the Timoshenko cantilever beam. The results are compared with the exact solution and the popular Meshless Local Petrov-Galerkin (MLPG). Optimal results have been obtained.

\section{MODELLING STRATEGY}

Let $\Omega$ be the domain of a body and $\Gamma$ its boundary subdivided in $\Gamma_{u}$ and $\Gamma_{t}$ that is $\Gamma=\Gamma_{t} \cup \Gamma_{u}$, as represented in Fig. 1. The general fundamental boundary value problem of linear elastostatics aims to determine the distribution of stresses $\boldsymbol{\sigma}$, strains $\boldsymbol{\varepsilon}$ and displacements $\boldsymbol{u}$, throughout the body, when it has constrained displacements $\overline{\boldsymbol{u}}$, on $\Gamma_{u}$ and is loaded by an external system of distributed surface and body forces with densities denoted, respectively by $\overline{\boldsymbol{t}}$, on $\Gamma_{t}$ and $\boldsymbol{b}$, in $\Omega$.

In the domain of the body, loaded by a system of external distributed surface and body forces with densities denoted, respectively by $\overline{\boldsymbol{t}}$, on the boundary $\Gamma_{t}$ and $\boldsymbol{b}$, in the domain $\Omega$, consider a statically admissible stress field $\boldsymbol{\sigma}$, that is any stress field that satisfies equilibrium with the system of applied external forces which therefore satisfies

$$
\boldsymbol{L}^{T} \boldsymbol{\sigma}+\boldsymbol{b}=\mathbf{0},
$$

in the domain $\Omega$, with boundary conditions

$$
\boldsymbol{t}=\boldsymbol{n} \boldsymbol{\sigma}=\overline{\boldsymbol{t}},
$$

on the static boundary $\Gamma_{t}$, in which $\boldsymbol{L}$ is a matrix differential operator; $\boldsymbol{t}$ is the vector of the traction components; $\overline{\boldsymbol{t}}$ is the vector of the prescribed tractions and $\boldsymbol{n}$ is the matrix of the components of the unit outward normal to the boundary.

In the domain $\Omega$, with boundary $\Gamma=\Gamma_{u} \cup \Gamma_{t}$, consider an arbitrary local domain $\Omega_{Q}$, assigned to a reference point $Q \in \Omega_{Q}$, with local boundary $\Gamma_{Q}=\Gamma_{Q i} \cup \Gamma_{Q t} \cup \Gamma_{Q u}$, in which $\Gamma_{Q i}$ is the interior local boundary, while $\Gamma_{Q t}$ and $\Gamma_{Q u}$ are local boundaries that share the global boundaries, respectively the static boundary $\Gamma_{t}$ and the kinematic boundary $\Gamma_{u}$, as represented

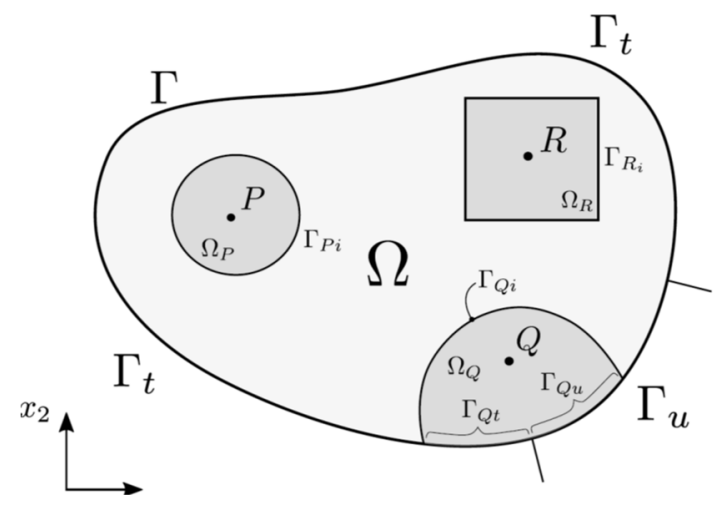

Figure 1: Schematic representation of the domain $\boldsymbol{\Omega}$ of the body. 
in Fig. 1. The work theorem is derived as a local form that is valid in an arbitrary domain $\Omega_{Q}$, associated to the reference point $Q$.

The general work theorem of the theory of structures establishes an energy relationship, valid in an arbitrary local domain $\Omega_{Q} \cup \Gamma_{Q} \in \Omega \cup \Gamma$, between two independent elastic fields that can be defined in the body which are, respectively a statically admissible stress field that satisfies equilibrium with a system of external distributed surface and body forces, and a kinematically admissible strain field that satisfies compatibility with a set of constrained displacements.

Expressed as an integral local form, defined in the local domain $\Omega_{Q} \cup \Gamma_{Q}$, the work theorem can be written in a compact form, simply as

$$
\int_{\Gamma_{Q}} \boldsymbol{t}^{T} \boldsymbol{u}^{*} d \Gamma+\int_{\Omega_{Q}} \boldsymbol{b}^{T} \boldsymbol{u}^{*} d \Omega=\int_{\Omega_{Q}} \boldsymbol{\sigma}^{T} \varepsilon^{*} d \Omega,
$$

in which the stress field $\boldsymbol{\sigma}$ and the strain field $\boldsymbol{\varepsilon}^{*}$ are not linked by any constitutive relationship and therefore, they are independent of each other.

Kinematic formulations consider, in the work theorem, a particular and convenient specification of the kinematically admissible strain field, leading thus to an equation of mechanical equilibrium that is used in numerical models, to generate the respective stiffness matrix of each model. A simple case of local equilibrium equations, based on a kinematically admissible strain field generated by a rigid-body displacement, is presented here.

Bearing in mind the key feature of the work theorem, which is the complete independence of the statically admissible stress field $\boldsymbol{\sigma}$ and the kinematically admissible strain field $\boldsymbol{\varepsilon}^{*}$, the strain field can be conveniently defined by a rigid-body displacement that is

$$
\boldsymbol{u}^{*}(\boldsymbol{x})=\boldsymbol{c},
$$

where $\boldsymbol{c}$ is a constant vector that conveniently leads to null strains that is

$$
\varepsilon^{*}(x)=\mathbf{0} .
$$

When the kinematically admissible strain field generated by the arbitrary rigid-body displacement (4) is considered, the local form of the work theorem, eqn (3), simply leads to the equation

$$
\int_{\Gamma_{Q}-\Gamma_{Q t}} \boldsymbol{t} d \Gamma+\int_{\Gamma_{Q t}} \overline{\boldsymbol{t}} d \Gamma+\int_{\Omega_{Q}} \boldsymbol{b} d \Omega=\mathbf{0},
$$

which states an integral form of mechanical equilibrium, of tractions and body forces, in the local domain $\Omega_{Q} \cup \Gamma_{Q}$.

The modelling strategy adopted in this paper, is based in the application of the work theorem, in the set of kinematically admissible strain fields, to solve the actual elastic problem.

Consider the local form of the work theorem, eqns (3), (6). To derive the equilibrium equations of the numerical model, the kinematic formulation of the local form is carried out through the specification of an appropriate kinematically admissible strain field $\boldsymbol{\varepsilon}^{*}$. This paper considers the arbitrary rigid-body displacement formulation that leads to the local form of equilibrium (eqn (6)). which are then used to generate the stiffness matrix of the meshfree numerical model.

The statically admissible stress field $\boldsymbol{\sigma}$, which is required to satisfy equilibrium with a system of external forces, is assumed as the stress field that settles in the body, when it is 
loaded by the actual system of external distributed surface and body forces, with the actual displacement constraints.

Recall that the elastic field that settles in the body is the only totally admissible elastic field that satisfies the given problem. Therefore, besides satisfying static admissibility, through eqns (1), (2), that is the same as satisfying equilibrium through eqn (6), generated by the weak form (3) of the work theorem, this unique totally admissible elastic field also satisfies kinematic admissibility defined as

$$
\varepsilon=L u,
$$

in the domain $\Omega$, with boundary conditions

$$
\boldsymbol{u}=\overline{\boldsymbol{u}},
$$

on the kinematic boundary $\Gamma_{u}$, in which the displacement $\boldsymbol{u}$ is assumed continuous with small derivatives, to allow for geometrical linearity of the strain field $\boldsymbol{\varepsilon}$. Hence, eqn (8), which specifies the constraints of the actual unique solution of the elastic problem must be fulfilled.

For the sake of simplicity, this paper considers the formulation of the meshfree numerical methods in the absence of body forces. Consequently, the equations of equilibrium are always defined only on the boundary of the local domain.

The essential feature of meshfree numerical methods is that they perform the discretization of the problem domain and boundaries with a set of scattered field nodes that do not require any mesh for the approximation of the field variables. The meshfree method ILMF, presented in this paper, is based on the moving least-squares (MLS) approximation.

Each node of the meshfree discretization is associated with its local domain. In general, this local domain is a circular or rectangular region, centered at the respective node, where the rigid-body displacement formulation of the work theorem is defined as a local form of mechanical equilibrium.

The local character of the MLS approximation is a consequence of the compact support of each node, where the respective shape functions are defined. The size of the compact support sets out, in a neighborhood of a sampling point, the respective domain of MLS approximation at this point. The domain of definition contains all the nodes whose MLS shape functions do not vanish at this sampling point. Therefore, the domain of influence of each node is the union of the MLS domains of all points in the node's local domain.

Finally, local meshfree formulations use a node-by-node stiffness calculation to generate, in the domain of influence of the local node, the respective rows of the global stiffness matrix.

In the absence of body forces, the local form of the work theorem eqn (6), can be written simply as

$$
\int_{\Gamma_{Q}-\Gamma_{Q t}} \boldsymbol{t} d \Gamma=-\int_{\Gamma_{Q t}} \overline{\boldsymbol{t}} d \Gamma,
$$

which represents mechanical equilibrium of the boundary tractions of the local domain $\Omega_{Q}$, associated with the field node $Q \in \Omega_{Q}$.

General numerical methods can be effectively formulated through a reduced integration of the equilibrium eqn (9) which, in the simplest linear case, leads to a point-wise discrete, form that improves the accuracy and the computational efficiency, as numerical results clearly demonstrate.

Hence, when a linear variation of tractions is assumed along each boundary segment of the local domain, the local integral form of equilibrium can be exactly evaluated with a single quadrature point, centered on each segment of the boundary. Eqn (9) then simply leads to 


$$
\frac{L_{i}}{n_{i}} \sum_{j=1}^{n_{i}} \boldsymbol{t}_{\boldsymbol{x}_{j}}=\frac{L_{t}}{n_{t}} \sum_{k=1}^{n_{t}} \overline{\boldsymbol{t}}_{\boldsymbol{x}_{k}}
$$

in which $n_{i}$ and $n_{t}$ denote the total number of integration points, or segments, defined on, respectively the interior local boundary $\Gamma_{Q}=\Gamma_{Q i} \cup \Gamma_{Q t} \cup \Gamma_{Q u}$, with length $L_{i}$, and the local static boundary $\Gamma_{Q t}$, with length $L_{t}$. This integrated equation obviously represents a pointwise discrete form of mechanical equilibrium of boundary tractions, evaluated at a set of points on the boundary of the local domain $\Omega_{Q}$.

Consider a meshfree discretization of the body. Then, the local meshfree method with linear reduced integration, symbolically referred to as ILMF, is used to compute the respective system of algebraic equations, in a node-by-node process, throughout traction evaluation at each central point of the boundary segments of the corresponding integrated local form (10) assigned to each node, with rectangular or circular local domains. Fig. 2 schematically represents these local domains with four segments and one integration point on each side, or quadrant, of the respective local domain.

Discretization of the integrated local form (10) is carried out with the MLS approximation, in terms of the unknown nodal parameters $\widehat{\boldsymbol{u}}$, thus leading to the system of two linear algebraic equations

$$
\frac{L_{i}}{n_{i}} \sum_{j=1}^{n_{i}} \boldsymbol{n}_{\boldsymbol{x}_{j}} \boldsymbol{D} \boldsymbol{B}_{\boldsymbol{x}_{j}} \widehat{\boldsymbol{u}}=\frac{L_{t}}{n_{t}} \sum_{k=1}^{n_{t}} \overline{\boldsymbol{t}}_{\boldsymbol{x}_{k}}
$$

that can be written as

$$
\boldsymbol{K}_{Q} \widehat{\boldsymbol{u}}=\boldsymbol{F}_{Q},
$$

in which $\boldsymbol{K}_{Q}$, the nodal stiffness matrix associated with the field node $Q$, is a $2 x 2 n$ matrix ( $n$ is the number of nodes included in the domain of influence of the reference node $Q$ that is the union of the MLS domains of definition of all integration points in the local domain $\Omega_{Q}$ ) given by

$$
\boldsymbol{K}_{Q}=\frac{L_{i}}{n_{i}} \sum_{j=1}^{n_{i}} \boldsymbol{n}_{\boldsymbol{x}_{j}} \boldsymbol{D} \boldsymbol{B}_{\boldsymbol{x}_{j}},
$$

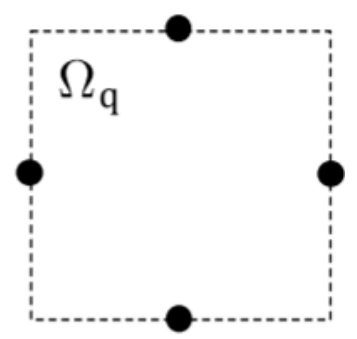

Figure 2: Schematic representation of rectangular domain, with one integration point on each edge of the local domain, for the computation of the local form of ILMF (10). 
and $\boldsymbol{F}_{Q}$ is the respective force vector given by

$$
\boldsymbol{F}_{Q}=\frac{L_{t}}{n_{t}} \sum_{k=1}^{n_{t}} \overline{\boldsymbol{t}}_{\boldsymbol{x}_{k}}
$$

Consider that the problem has a total of $N$ field nodes $Q$, each one associated with the respective local domain $\Omega_{Q}$. Assembling eqn (9) for all $M$ interior and static-boundary field nodes leads to the global system of $2 M \times 2 N$ equations

$$
\boldsymbol{K} \widehat{\boldsymbol{u}}=\boldsymbol{F} .
$$

Finally, the remaining equations are obtained from the $N-M$ boundary field nodes on the kinematic boundary. For a field node on the kinematic boundary, a direct interpolation method is used to impose the kinematic boundary condition as

$$
\boldsymbol{u}_{k}=\boldsymbol{\Phi}_{k} \widehat{\boldsymbol{u}}=\overline{\boldsymbol{u}}_{k}
$$

with $k=1.2$, where $\overline{\boldsymbol{u}}_{k}$ is the specified nodal displacement component. Eqn (16) are directly assembled into the global system of eqn (15).

It can be easily anticipated high computational efficiency, with very accurate results, of this local formulation with linear reduced integration. As a matter of fact, the nodal stiffness matrix is effectively computed, in eqn (13), with only 4 integration points ( 1 integration point on each side of the local boundary), which basically implies a very short processing time to run the analysis. In addition, the reduced integration leads to high accuracy of the results, which plays a key role in the behavior of ILMF, since it implies a reduction of the nodal stiffness which, in turn, leads to an increase of the solution accuracy and, which is most important, presents no instabilities.

\section{NUMERICAL EXAMPLES}

This section presents some numerical results for Cantilever beam for three different nodal configurations. The effects regular and irregular nodal arrangement are analyzed and compared with exact solution and other popular meshfree method (MLPG).

For a generic node $i$, the size of the local support $\Omega_{S}$ and the local domain of integration $\Omega_{q}$ are respectively given by

$$
\begin{aligned}
& r \Omega_{S}=\alpha_{S} d_{i}, \\
& r \Omega_{q}=\alpha_{q} d_{i},
\end{aligned}
$$

in which $d_{i}$ represents the distance of the node $i$, to the nearest neighboring node; for the analysis is performed for one value of the local support domain size $\left(\alpha_{S}=2.13\right)$, and the local quadrature domain size $\left(\alpha_{q}=0.5\right)$.

The nodal irregularity is generated by changing randomly the coordinates of the nodal regularity distribution by small distance, this movement can be calculated by

$$
\begin{gathered}
x_{1 i}^{\prime}=x_{1 i} \pm c_{n} d_{x_{1 i},} \\
x_{2 i}^{\prime}=x_{i} \pm c_{n} d_{x_{2 i}},
\end{gathered}
$$

in which $C_{n}$ is a parameter that controls the nodal irregularity and varies randomly in the range of 0.0 and 0.4 . For nodes located in the boundary there are restrictions that depend on the position of the node. 
Displacement and energy norms can be used for error estimation and can be computed respectively as

$$
\begin{gathered}
\|\boldsymbol{u}\|=\left[\int_{\Omega} \boldsymbol{u}^{T} \boldsymbol{u} d \Omega\right]^{\frac{1}{2}}, \\
\|\varepsilon\|=\left[\frac{1}{2} \int_{\Omega} \varepsilon^{T} \mathbf{D} \varepsilon d \Omega\right]^{\frac{1}{2}} .
\end{gathered}
$$

The relative error for $\|u\|$ and $\|\varepsilon\|$ is given, respectively by

$$
\begin{gathered}
r_{u}=\frac{\left\|\boldsymbol{u}_{\text {num }}-\boldsymbol{u}_{\text {exact }}\right\|}{\left\|\boldsymbol{u}_{\text {exact }}\right\|}, \\
r_{\varepsilon}=\frac{\left\|\varepsilon_{\text {num }}-\varepsilon_{\text {exact }}\right\|}{\left\|\varepsilon_{\text {exact }}\right\|} .
\end{gathered}
$$

A cantilever beam, as shown in Fig. 3, is subjected to a parabolic traction at the free end. The main properties are tabulated in Table 1 and the problem is solved for plane stress case.

The parabolic traction and the moment of inertia are given by

$$
\begin{gathered}
\bar{t}_{2}\left(x_{2}\right)=-\frac{P}{2 I}\left(\frac{D^{2}}{4}-x_{2}^{2}\right), \\
I=\frac{D^{3}}{12} .
\end{gathered}
$$

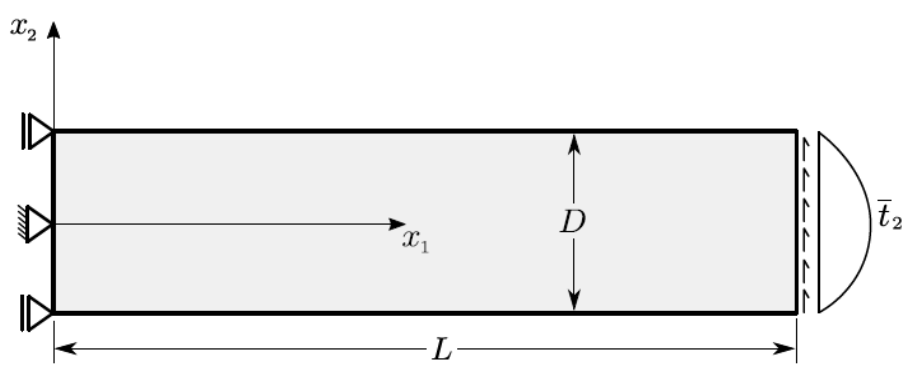

Figure 3: Cantilever beam.

Table 1: Properties of the cantilever beam.

\begin{tabular}{lc}
\hline Parameters & Values \\
\hline Height, D & $12(\mathrm{~m})$ \\
Length, L & $48(\mathrm{~m})$ \\
Thickness, t & $1(\mathrm{~m})$ \\
Load, P & $1000(\mathrm{~N})$ \\
Modulus of elasticity, E & $30(\mathrm{GPa})$ \\
Poisson's ratio, $v$ & 0.3 \\
\hline
\end{tabular}


The equations for the exact displacement are:

$$
\begin{gathered}
u_{1}\left(x_{1}, x_{2}\right)=-\frac{P x_{2}}{6 E I}\left[\left(6 L-3 x_{1}\right) \cdot x_{1}+(2+v)\left(x_{2}^{2}-\frac{D^{2}}{4}\right)\right], \\
u_{2}\left(x_{1}, x_{2}\right)=-\frac{P x_{2}}{6 E I}\left[3 v x_{2}^{2}\left(L-x_{1}\right)+(4+5 v) \frac{D^{2} x_{1}}{4}+\left(3 L-x_{1}\right) x_{1}^{2}\right] .
\end{gathered}
$$

And the exact stress components are given by

$$
\begin{gathered}
\sigma_{11}\left(x_{1}, x_{2}\right)=-\frac{P\left(L-x_{1}\right) x_{2}}{I}, \\
\sigma_{12}\left(x_{1}, x_{2}\right)=-\frac{P}{2 I}\left(\frac{D^{2}}{4}-x_{2}^{2}\right), \\
\sigma_{22}\left(x_{1}, x_{2}\right)=0 .
\end{gathered}
$$

The ILMF is used for solving this problem. Both a regular $\left(C_{n}=0\right)$ and irregular $\left(C_{n}=0.1,0.2,0.3\right.$ and 0.4$)$ nodal distribution are employed with a discretization of $21 \times 9=$ 189 nodes.

In the first discretization, Fig. 4, the nodes located in the boundary have a regular distribution and nodes located inside the beam have irregular distribution for the solution using MLPG [15].

The energy relative error for two different methods are presented in Fig 6. The energy relative error for MLPG and ILMF have the same expressions presented in eqns (22), (24). These values for MLPG were obtained in [15].

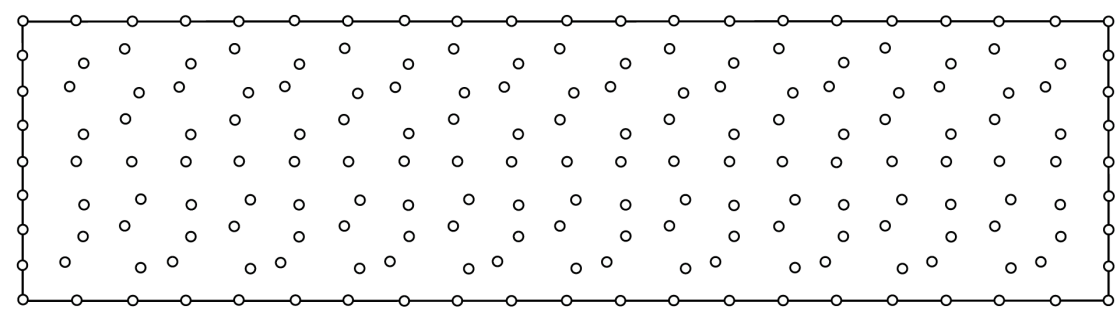

Figure 4: Irregular internal nodal arrangement for the cantilever beam (MLPG) [15].

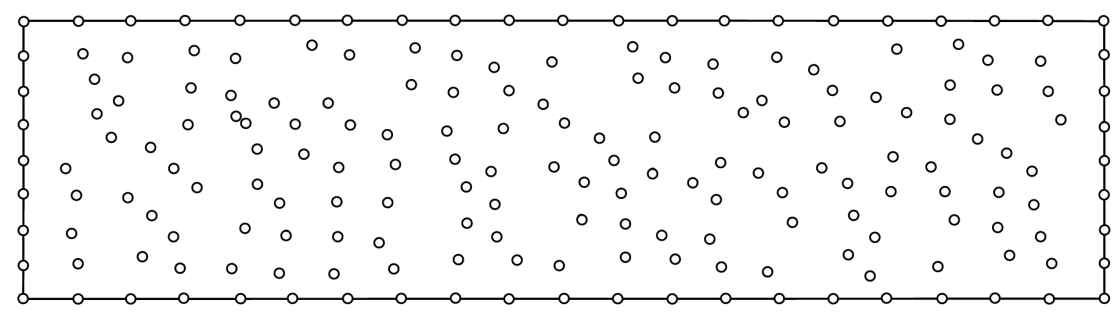

Figure 5: Irregular internal nodal arrangement (ILMF). 


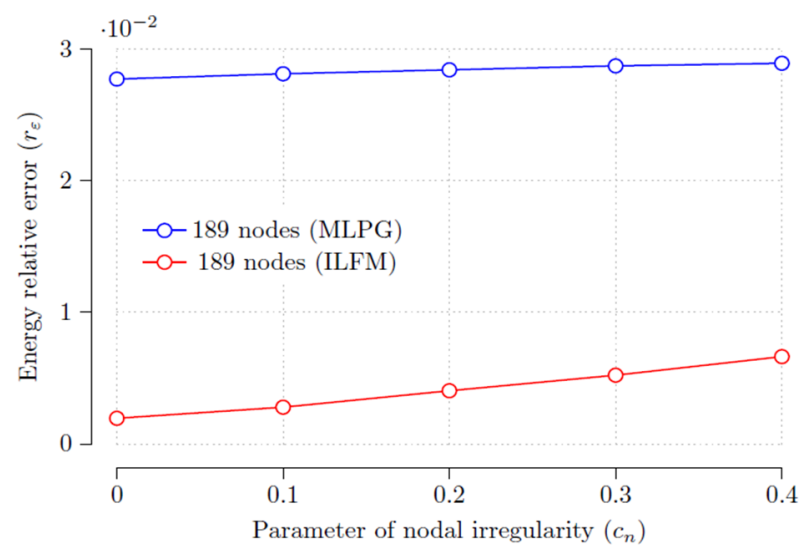

Figure 6: Effect of irregularity on the nodal arrangement on energy relative error for MLPG and ILMF.

Fig. 6 shows that the energy relative error for the irregular nodal discretization, using ILMF and MLPG presented similar behavior for the discretization, but the ILMF presented more accuracy.

Other different irregular nodal discretization was carried out to know the influence on the accuracy of the energy and displacement relatives errors. Two additional configurations are presented with $11 \times 5=55$ nodes and $33 \times 17=561$ nodes.

Figs 7 and 8 illustrates the variation of energy and displacement relative error as a function of the size of the nodal irregularity parameter, which varies between 0.0 to 0.4 , with 0.1 increments. Results are presented for three nodal discretization using values fixed of the local support domain $\left(\alpha_{\mathrm{s}}=2.13\right)$ and the local quadrature domain $\left(\alpha_{\mathrm{q}}=0.5\right)$, using both nodal configurations.

Figs 7 and 8 show that the energy and displacement relative error decreases with finer nodal distributions for both configuration. This result evidences that the meshless methods with reduce integration presents an optimal behavior when the mesh is refined.

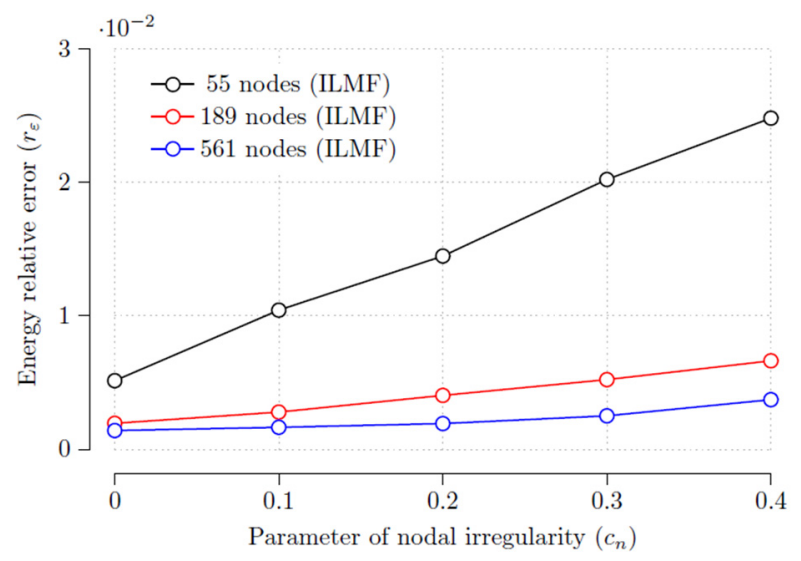

Figure 7: Effect of irregularity on the nodal arrangement on energy relative error for ILMF. 


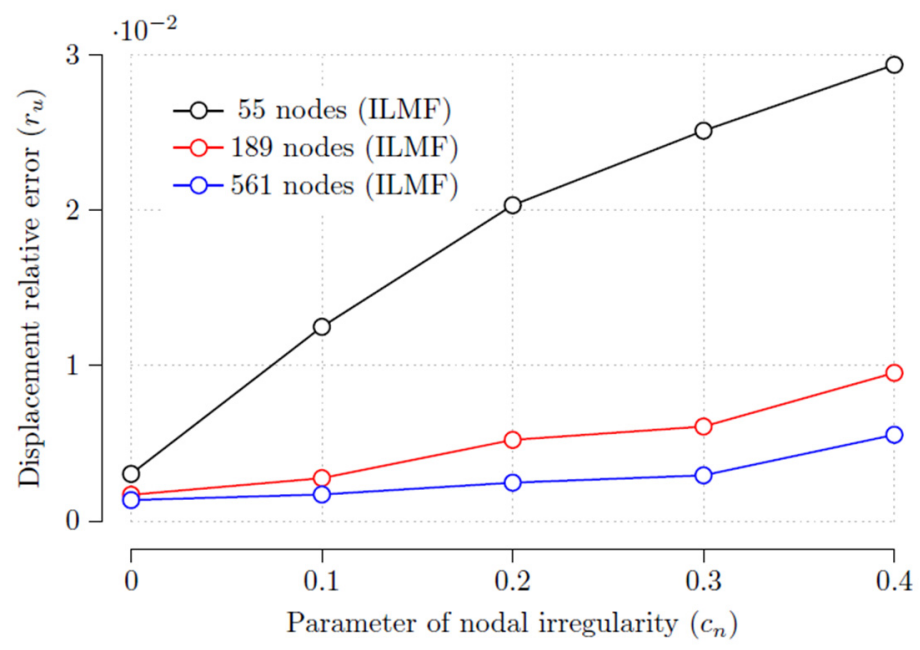

Figure 8: Nodal arrangement irregularity effect on displacement relative error for ILMF.

\section{CONCLUSIONS}

The effect of the nodal irregularity is irrelevant on energy and displacement relative errors for different configurations and discretization on ILMF. This fact reveals that the meshfree method with reduced integration (ILMF) and meshfree Local Petrov-Galerkin (MLPG) are stable for irregular nodal arrangements, but ILMF shows better accuracy.

The presented results prove that MLPG and ILMF are suitable for solving problems with irregular nodal arrangement. Both are accurate, but ILMF is more precise. Even with $C_{n}=$ 0.4 , the maximum irregularity used in this analysis, the ILMF presents a better precision than MLPG using the same discretization.

The results of the energy and displacement relative error for different nodal discretization for the cantilever beam, using ILMF and MLPG, confirm that the effect of the nodal irregularity is very small, and can be neglected.

The small errors obtained for different nodal discretization with a large parameter of irregular arrangement $\left(C_{n}=0.4\right)$, allow the application of this technique to realistic engineering problems involving inhomogeneous irregular geometries.

\section{ACKNOWLEDGEMENTS}

The program "PECC - Pós Graduação em Estruturas e Construção Civil", Department of Civil and Environmental Engineering, Faculty of Technology, University of Brasília. CNPq - Brazilian National Counsel of Technological and Scientific Development for Wilber's PhD scholarship. "FAP/DF - Fundação de Apoio à Pesquisa do Distrito Federal" for financial support.

\section{REFERENCES}

[1] Daxíni, S.D. \& Prajapati, J.M., A review on recent contribution of the meshfree methods to structure and fracture mechanics applications. The Scientific Word Journal, 2014, pp. 1-13, 2014. DOI: 10.1155/2014/247172.

[2] Finalyson, B.A., The Method of Weighted Residuals and Vibrational Principles, Academic Press, 1972. 
[3] Chen, J.S., Hilhnan, M. \& Chi, S.W., Meshfree Methods: Progress made after 20 years. Journal of Engineering Mechanics, 143(4), p. 04017001, 2017.

DOI: $10.1061 /$ (asce)em.1943-7889.0001176.

[4] Nayroles, B., Touzot, G. \& Villon, P., Generalized the Finite Element Method: Diffuse Approximation and Diffuse Elements. Computational Mechanics, 10(5), pp. 307-318, 1992. DOI: $10.1007 / \mathrm{bf00364252.}$

[5] Li, W.K., Jun, S. \& Zhang, Y.F., Reproducing kernel particle methods. International Journal for Numerical Methods in Engineering, 20, pp. 1081-1106, 2007.

[6] Belytshko, T., Lu, Y.Y. \& Gu, L., Element free Galerkin methods. International Journal for Numerical Methods in Engineering, 37(2), pp. 229-256, 1994.

[7] Atluri, S.N. \& Zhu, T., A new Meshless Local Petrov-Galerkin (MLPG) approach in computational mechanics. Computational Mechanics, 22(2), pp. 117-127, 1998. DOI: $10.1007 / \mathrm{s} 004660050346$.

[8] Atluri, S.N. \& Shen, S., The meshless local Petrov-Galerkin (MLPG) method: A simple and less-costly alternative to the finite element and boundary element methods. CMES: Computer Modelling in Engineering and Sciences, 3(1), pp. 11-15, 2002.

[9] Zhu, T., Zhang, J. \& Atluri, S.N., A local boundary integral equation (LBJE) method in computational mechanics and a meshless discretization approach. Computational Mechanics, 21(3), pp. 223-235, 1998. DOI: 10.1007/s004660050297.

[10] Liu, G.R. \& Gu, Y.T., A local point interpolation method for stress analysis of twodimensional solids. Structural Engineering and Mechanics, 11(2), pp. 221-236, 2001. DOI: 10.12989/sem.2001.11.2.221.

[11] Liu, G.R., Yan, L., Wang, 1. G. \& Gu, Y.T., Point interpolation method based on local residual formulation using radial basis functions. Structural Engineering and Mechanics, 14(6), pp. 713-732, 2002. DOI: 10.12989/sem.2002.14.6.713.

[12] Atluri, S.N., Han, Z.D. \& Rajendran, A.M., A new implementation of the meshless finite volume method through the mlpg mixed approach. CMES: Computer modelling in Engineering and Sciences, 6, pp. 491-513, 2004.

[13] Oliveira, T. \& Portela, A., Weak-form-collocation: A local meshless method in linear elasticity. Engineering Analysis with Boundary Elements, 73, pp. 144-160, 2016. DOI: 10.1016/j.enganabound.2016.09.010.

[14] Santana, E., Oliveira, T., Vélez, W. \& Portela, A., Meshfree method with reduced integration and automatic parameter optimization. Computers and Structures, 2017.

[15] Liu, G.R., Meshfree Methods Moving Beyond The Finite Element Method, CRC Press, 2003. 